\title{
Value of contrast-enhanced ultrasound area ratio in identifying benign and malignant small breast masses
}

\author{
Wei Wang", Yan Zheng", Xiao-Feng Wü, Dan Zhao, Li-Zhu Hou, Fei Shi, Jin-Jin Liu, Feng-Lin Dong \\ Department of Ultrasound, the First Affiliated Hospital of Soochow University, Suzhou, China \\ Contributions: (I) Conception and design: FL Dong, W Wang, Y Zheng; (II) Administrative support: FL Dong; (III) Provision of study materials or \\ patients: W Wang, XF Wu, D Zhao, LZ Hou; (IV) Collection and assembly of data: W Wang, F Shi, JJ Liu; (V) Data analysis and interpretation: W \\ Wang; (VI) Manuscript writing: All authors; (VII) Final approval of manuscript: All authors. \\ \#These authors contributed equally to this work. \\ Correspondence to: Feng-Lin Dong. Department of Ultrasound, the First Affiliated Hospital of Soochow University, Suzhou, China. \\ Email: fldong@suda.edu.cn.
}

Background: Tumor size affects clinical staging and is closely related to prognosis. Therefore, early diagnosis of breast cancer is one of the most important methods to reduce mortality and improve prognosis. However, minimal breast cancer is difficult to differentiate from small benign breast masses due to insufficient typical malignant signs. The significantly increased range of enhancement can be an important indication for the prediction of malignancy; however, quantitative studies on the extent of enhancement are rarely reported. The purpose of this study was to investigate the value of contrast-enhanced ultrasound (CEUS) area ratio in finding benign and malignant small breast masses.

Methods: A retrospective analysis was conducted on 88 patients with breast masses confirmed by surgery or needle biopsy (the maximal diameter not over $1 \mathrm{~cm}$ ). 88 breast masses were divided into the younger age group (not over 40 years old) and older age group (over 40 years old) according to the patient's age. The receiver operating characteristic (ROC) curve was used to determine the cutoff values of CEUS area ratio in diagnosing benign or malignant small breast masses in each group. The efficiency of different cutoff values in finding benign and malignant small breast masses of the distinct groups was analyzed.

Results: The CEUS area ratio of malignant mass was larger than benign masses $(\mathrm{P}<0.05)$. The CEUS area ratio of malignant masses in the younger age group was larger than that in the older age group $(\mathrm{P}<0.05)$. The results of the ROC curve analysis showed that the area under the curve (AUC) and the cutoff values of the entire group, the younger age group, and the older age group were 0.887, 1.65; 0.909, 1.95; and 0.908, 1.22 , respectively. When the cutoff value of the older age group was reduced from 1.65 to 1.22 , its diagnostic sensitivity was improved significantly $(\mathrm{P}<0.05)$.

Conclusions: CEUS area ratio has specific application value in finding benign and malignant small breast masses. Proper reduction of the cutoff value of elderly patients can further improve its diagnostic sensitivity without significantly reducing the specificity.

Keywords: Minimal breast mass; contrast-enhanced ultrasound (CEUS); area ratio

Submitted Jul 15, 2020. Accepted for publication Sep 29, 2020.

doi: 10.21037/gs-20-697

View this article at: http://dx.doi.org/10.21037/gs-20-697 


\section{Introduction}

Breast cancer remains the most common cancer and the leading cause of cancer mortality in women worldwide (1). How to prevent is unknown; thus, early detection and early diagnosis of breast cancer is of great importance. Ultrasound has the advantages of nonradiation, low price, convenience and high diagnostic accuracy. Meanwhile most Chinese women have relatively small, dense breasts (2). Therefore, ultrasound was chosen as the primary imaging modality for breast examination (3). Minimal breast cancer refers to breast cancer whose diameter is less than $10 \mathrm{~mm}$ (4), and it is difficult to differentiate it from benign small breast tumors by conventional ultrasound diagnosis because of insufficient typical malignant signs (5). Contrast-enhanced ultrasound (CEUS) is a new ultrasound technology that can display tumor microcirculation in real-time. It is widely used in the differential diagnosis of benign and malignant breast masses. However, the observation indicators of each research morality are not the same. Balleyguier et al. (6) stated that when there are some characteristics, such as rapid enhancement, peripheral ring enhancement and obvious expansion of enhancement range, it is suggested that the lesion is malignant. Zhao et al. (7) found out Lesions showing claw-shape enhancement and contrast medium persistence were classified as malignant, and lesions showing homogeneous enhancement without contrast persistence were classified as benign. It shows excellent diagnostic efficiency as one sign of a malignant tumor, the significantly increased range of enhancement after CEUS can be used as an essential clue to predict malignancy (8-10). However, there are few reports on the quantitative study of the enlargement range. The purpose of this study was to investigate the value of CEUS area ratio in the differential diagnosis of benign and malignant small breast masses by retrospectively analyzing the ultrasonic data of 88 cases of small breast masses. We present the following article in accordance with the STARD reporting checklist (available at http://dx. doi. org/10.21037/gs-20-697).

\section{Methods}

\section{Research subjects}

From July 2016 to July 2019, a total of 1,208 consecutive patients with breast masses were referred to our department for CEUS examinations. We excluded 1,056 cases with the maximum diameter over $1 \mathrm{~cm}, 59$ cases without pathological results and 5 cases with poor image quality. Finally, the data of 88 patients ( 88 breast masses) with the maximum diameter not over $1 \mathrm{~cm}$ who were confirmed by surgery or needle biopsy in our hospital were retrospectively analyzed. Conventional ultrasound and CEUS were performed 1-2 d before surgery or needle biopsy for all the masses, all of which were primary lesions and had received no treatment before. All the patients were female with an average age of $42.59 \pm 11.03$ (range, 24-83) years. They were divided into the younger age group (not over 40 years old) and the older age group (over 40 years old) with their age (11) (Figure 1). The study was reviewed and approved by the Medical Ethics Committee of the First Affiliated Hospital of Soochow University, with the written consent of all the patients taking part in the experiment. All procedures performed in this study involving human participants were in accordance with the Declaration of Helsinki (as revised in 2013).

\section{Instruments and methods}

Mindray Resona7, LOGIQ E9, and MyLab Class C Color Doppler sonography were used (with probe models of L11-3U; ML6-15, 9L; and LA523 and LA522, and with contrast agent of SonoVue (Bracco Corporate, Shanghai, China). Before angiography, all patients agreed and signed informed consent. The patient was placed in the supine position with both hands raised to expose the breast fully. First, grayscale ultrasonography scanned the mammary gland, and if any nodule was found, the characteristics of the nodule, including the position, maximum diameter, shape, edge, internal echo, and rear echo were recorded. Then the section of the nodule, with the most abundant blood supply, was selected for angiographic examination. After $3.0 \mathrm{~mL}$, SonoVue was injected through the cubital vein mass, $5 \mathrm{~mL}$ normal saline was rapidly injected for flushing, and a timer and dynamic storage function were started while the contrast agent was injected. During the examination, we asked the patient to breathe calmly, kept the selected section unchanged, and did not press the probe.

\section{Image analysis}

Two experienced senior physicians tested all ultrasound images without knowledge of the patient's clinical data. When the two did not agree, a consensus was reached 


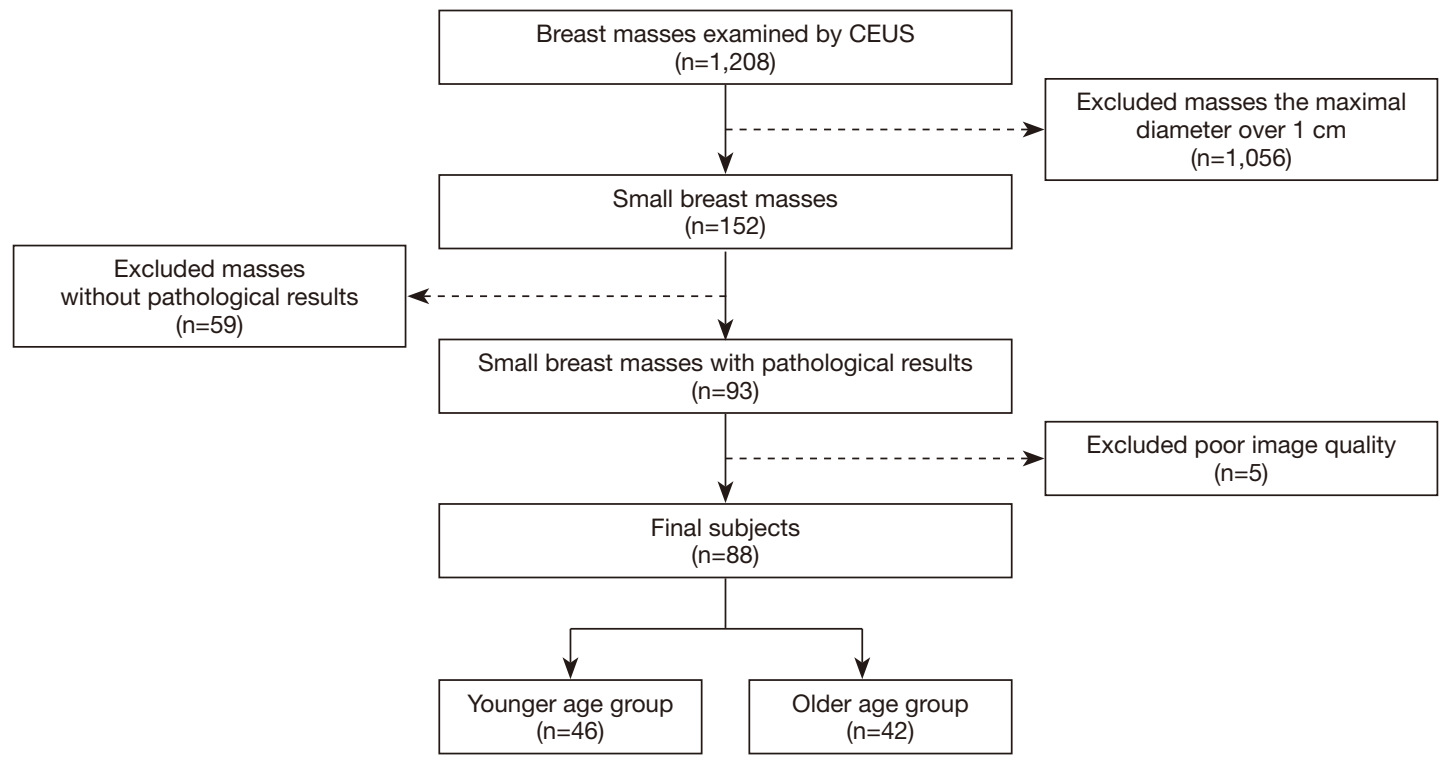

Figure 1 Flow diagram of participants. CEUS, contrast-enhanced ultrasound.

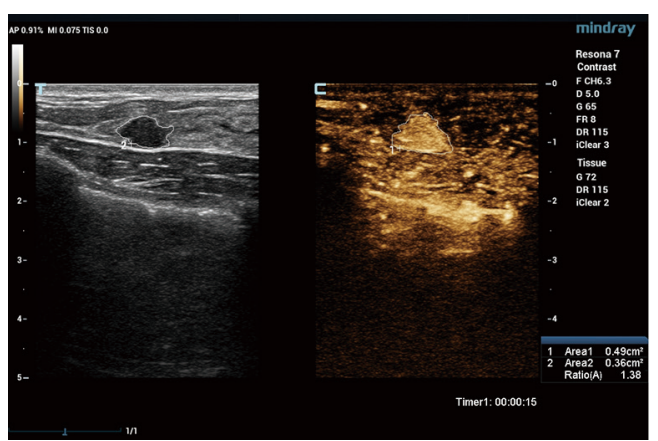

Figure 2 Ultrasonography of fibroadenoma of breast. The picture came from a 28-year-old female patient and showed a hypoechoic mass in the left breast on ultrasound. The maximum diameter of the mass was $0.9 \mathrm{~cm}$ under conventional ultrasound, and the contrast area ratio was 1.38

through discussion. The dynamic contrast images stored in the hard disk of the instrument were played back, and the images were frozen when the contrast intensity of the nodule reached its peak. The grayscale image and the contrast image of the nodule were tracked, respectively (Figure 2). The area ratio before and after contrast was calculated, and the average value was taken after repeated measurements for three times. When the nodule displayed iso-enhancement with the surrounding tissue, and the boundary was unclear, the default area ratio before and after nodule contrast was 1 .

\section{Statistical analysis}

The SPSS 23.0 software package was used for statistical analysis of the data. The normality of measurement data was checked with the K-S test. Measurement data of normal distribution are expressed as mean \pm standard deviation $(\bar{x} \pm s)$ and measurement data of non-normal distribution as median (interquartile range) [M (P25, P75)]. The MannWhitney $\mathrm{U}$ test was used for the comparison between two independent samples. The receiver operating characteristic (ROC) curve was constructed from the pathological results, the area under the curve (AUC) was obtained, and the diagnostic threshold was calculated. $\chi^{2}$-test was used to compare the diagnostic performance between different diagnostic thresholds. $\mathrm{P}<0.05$ was considered statistically significant.

\section{Results}

\section{Pathological results}

Among the 88 breast masses, 54 cases were benign mass, including 29 cases of fibroadenoma, 17 cases of adenopathy, 6 cases of intraductal papilloma, and 2 cases of plasma cell mastitis. There were 29 cases of left lesion and 25 cases of 
Table 1 Differences in the contrast area ratios between benign and malignant masses among the groups [M (P25, P75)]

\begin{tabular}{lccc}
\hline Group & Number of cases & Contrast area ratio & Z value \\
\hline General group & 54 & $1.19(1.04,1.58)$ & -6.096 \\
Benign & 34 & $2.08(1.59,2.61)$ & \\
Malignant & & & -4.592 \\
Younger group & 29 & $1.27(1.03,1.63)$ & $<0.001$ \\
Benign & 17 & $2.44(2.01,2.96)$ & -4.447 \\
Malignant & & & $<0.001$ \\
Older group & 25 & $1.13(1.04,1.33)$ & \\
Benign & 17 & $1.77(1.32,2.24)$ & \\
Malignant & &
\end{tabular}

$\mathrm{P}<0.05$ is considered statistically significant.

Table 2 Difference however area ratio between benign and malignant masses in different age groups [M (P25, P75)]

\begin{tabular}{|c|c|c|c|c|}
\hline Group & Number of cases & Contrast area ratio & $Z$ value & $P$ value \\
\hline Younger group & 29 & $1.27(1.03,1.63)$ & & \\
\hline Older group & 25 & $1.13(1.04,1.33)$ & & \\
\hline Malignant & & & -2.705 & 0.007 \\
\hline Older group & 17 & $1.77(1.32,2.24)$ & & \\
\hline
\end{tabular}

$\mathrm{P}<0.05$ is considered statistically significant.

right lesion. Twelve cases showed breast mass, 22 cases had breast pain and 2 cases had nipple discharge. There were 34 cases of malignant mass, including 23 cases of invasive ductal carcinoma, 9 cases of ductal carcinoma in situ, 1 case of invasive lobular carcinoma, and 1 case of mucinous adenocarcinoma. There were 15 cases of left lesion and 19 cases of right lesion. Nine cases showed breast mass, 19 cases had breast pain and 3 cases had nipple discharge.

\section{Comparison of the contrast area ratio between benign and malignant masses in each group}

The differences in the contrast area ratios of benign and malignant masses among the general group, the younger age group, and the older age group were statistically significant $(\mathrm{P}<0.05)($ Table 1).

\section{Comparison of contrast area ratios between benign and malignant masses in different age groups}

There was no statistically significant difference between benign and malignant masses in different age groups $(\mathrm{P}>0.05)$ when benign and malignant masses were compared with age grouping. However, the contrast area ratio of malignant masses in the younger age group was higher than that in the older age group, with a statistically significant difference $(\mathrm{P}<0.05)$ (Table 2).

\section{ROC curve was used to evaluate the value of contrast area ratio in the diagnosis of benign and malignant breast masses in different groups}

When the contrast area ratio was taken as the test variable, 

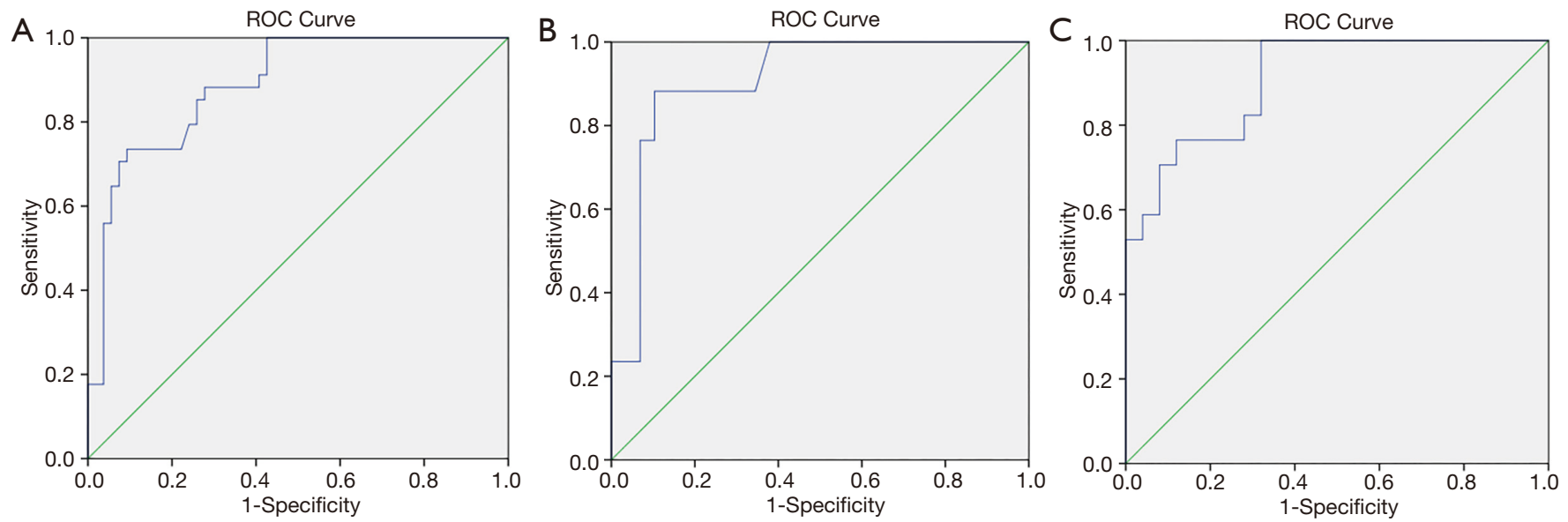

Figure 3 ROC curve of contrast area ratio for evaluating benign and malignant breast mass in each group. (A) The ROC curve of contrast area ratio for evaluating benign and malignant masses in the general group; (B) the ROC curve of contrast area ratio for evaluating the benign and malignant masses in the younger age group; $(\mathrm{C})$ the ROC curve of contrast area ratio for evaluating the benign and malignant masses in the older age group.

Table 3 The value of contrast area ratio in diagnosing benign and malignant breast masses in different groups is evaluated with ROC curve

\begin{tabular}{lcccccc}
\hline \multirow{2}{*}{ Group } & AUC & Standard deviation & P value & \multicolumn{2}{c}{$95 \%$ confidence interval } & Cutoff for diagnosis \\
\cline { 5 - 6 } & & & & Lower limit & Upper limit & \\
General group & 0.887 & 0.04 & $<0.001$ & 0.819 & 0.955 & 1.65 \\
Younger group & 0.909 & 0.05 & $<0.001$ & 0.821 & 0.996 & 1.95 \\
Older group & 0.908 & 0.04 & $<0.001$ & 0.823 & 0.993 & 1.22 \\
\hline
\end{tabular}

and the ROC curve was drawn with the pathological results as the gold standard (Figure 3), the results showed the contrast area ratio had a specific value in the differential diagnosis of each group (Table 3).

\section{The efficacy of pre- and post-adjusted cutoff value for differentiating benign from malignant breast masses in different age groups}

When 1.65 and 1.95 were respectively used as a cutoff value for diagnosis in the younger age group, the diagnostic efficacy was shown in Table 4. Before and after the adjustment of the cutoff value for diagnosis, the differences in sensitivity, specificity, positive predictive value, negative predictive value, and accuracy were not statistically significant $(\mathrm{P}>0.05)$ (Figure 4). When 1.65 and 1.22 were respectively taken as the cutoff value for diagnosis in the elder age group, the diagnostic efficacy was shown in Table 4. Reducing the cutoff value for diagnosis from 1.65 to
1.22 in the elder age group could significantly improve the diagnostic sensitivity $(\mathrm{P}<0.05)$ (Figure 5$)$. The specificity and positive predictive value decreased slightly, and the negative predictive value and diagnostic accuracy increased, but the differences were not statistically significant $(\mathrm{P}>0.05)$.

\section{Discussion}

Tumor size affects its clinical staging and is closely related to its prognosis, so early diagnosis of breast cancer is one of the most important methods to reduce mortality and improve the prognosis (12). When the diameter of a solid tumor reaches 1-2 $\mathrm{mm}$, tumor neovascularization can be observed (13), and the morphology and distribution characteristics of tumor neovascularization are different (14). CEUS can clearly show the microvascular perfusion inside and around the tumor. Relevant studies (8-10) suggested that the significantly increased range of tumor enhancement after angiography may be an important indication for the 
prediction of malignancy. However, in clinical work, the judgment of whether the enhanced range after angiography is significantly increased is easily affected by subjective factors and cannot be quantified, which brings difficulties to the diagnosis. Therefore, a quantitative index is urgently needed to reflect the changes objectively between enhancement after angiography.

After the nodules were grouped and analyzed, it was found that the contrast area ratio of the malignant tumor was higher than a benign tumor, and the difference was statistically significant. The difference is because benign tumors have less neovascularization and more uniform distribution. However, under the action of angiogenic factors, malignant tumors have more neovascularization, and most of these abnormal blood vessels are clustered at the edge of the tumor body (15). Simultaneously, some malignant tumors are often accompanied by intraductal carcinoma or breast adenopathy, which is often challenging to be shown by conventional ultrasound, but most of them show enhancement during angiography $(9,16)$. The results showed that there was no significant difference, whereas the area ratio between benign masses in different age groups. However, in malignant tumors, the contrast area ratio of the younger age group was significantly higher than the older age group, which was consistent with relevant studies $(11,17)$. It may be related to the fact that the breast cancer of the younger age group is usually poorly differentiated and more aggressive to the surrounding tissues (18).

In this study, it was found that for the breast masses in the older age group, appropriate reduction of the cutoff value could significantly improve the diagnostic sensitivity in evaluating benign and malignant breast masses with the contrast area ratio of CEUS. When ROC analysis was performed on the complete set of the data, the cutoff value for the diagnosis of contrast area ratio was 1.65 . When ROC analysis was performed on the older age group alone, the cutoff value for diagnosis was reduced to 1.22 , and then the diagnostic sensitivity increased from $58.8 \%$ to $94.1 \%$. Six malignant masses were misdiagnosed as benign masses when the original cutoff value was applied and correctly diagnosed as malignant masses when the new cutoff value was applied.

The application of the adjusted cutoff value significantly reduced the rate of missed diagnosis, but there were still 3 cases of malignant mass misdiagnosed as benign mass, including 2 cases of ductal carcinoma in situ and 1 case of invasive ductal carcinoma. Liberman et al. (19) proposed that some ductal carcinoma in situ of low-level could rely on healthy peripheral blood vessels to provide necessary nutrients at the early stages of the lesion without being accompanied by the formation of abnormal peripheral blood vessels. Simultaneously, for some malignant tumors, the immune system of the body can produce dynamic changes of reverse infiltration, forming an immune response zone around the lesion and inhibiting the formation of peripheral microvessels to a certain extent (20). In benign lesions, adenopathy and intraductal papilloma are more challenging, distinguishing, and prone to false positive. Breast adenopathy often shows lobular acinar hyperplasia accompanied by stromal hyperplasia. However, due to some target cells more sensitive to hormones, hyperplasia can appear in nodules under the action of hormones. Therefore, we hypothesize that "breast nodule" is only the apparent hyperplasia area observed by conventional ultrasound. Conventional ultrasonography often enhances ultrasound. Adenosis is often enhanced in CEUS. In the misdiagnosed intraductal papilloma, local atypical hyperplasia accompanies some cases. Jaffe et al. (21) considered that the ductal wall could be involved when the atypical hyperplasia was extended, resulting in the enlargement of the area after angiography.

At present, most of the study only requires a simple qualitative description of whether the enhanced range is significantly increased after angiography, and there are few reports on the quantitative study of the increased range. In this study, the value of CEUS area ratio in the differential diagnosis of small breast masses was analyzed, and the patients of different ages were further discussed in groups. Together, this study also has the following limitations: (I) the surgeons did not outline the edges of nodules the same during angiographic analysis. Deviation still existed, although the mean value was taken; (II) the results should be interpreted with caution due to the nature of the retrospective data and the relatively small sample size in the present study. Prospective studies with more cases should be carried out for verification and further quantification of the contrast-enhanced ultrasound area ratio in the predicting of malignancy; (III) because the number of cases of noninvasive cancer was limited, the subdivision might lead to the incredibility of the study results. Therefore, further grouping discussion of malignant tumors, according to pathological types, was not conducted.

To sum up, the contrast-enhanced ultrasonography area ratio in the differential diagnosis of benign and malignant breast small masses has specific application value. With 1.65 as the cutoff value, higher diagnostic efficiency can be 
Table 4 The efficacy of pre- and post-adjusted cutoff value for differentiating benign from malignant breast masses in different age groups (cases \%)

\begin{tabular}{lcccccc}
\hline Group & Cutoff value & Sensitivity & Specificity & Pos. value & Neg. value & Precision \\
\hline Younger group & 1.65 & $88.2(15 / 17)$ & $86.2(25 / 29)$ & $78.9(15 / 19)$ & $92.6(25 / 27)$ & $87(40 / 46)$ \\
& 1.95 & $88.2(15 / 17)$ & $89.7(26 / 29)$ & $83.3(15 / 18)$ & $92.9(26 / 28)$ & $89.1(41 / 46)$ \\
Older group & 1.65 & $58.8(10 / 17)$ & $92(23 / 25)$ & $83.3(10 / 12)$ & $76.6(23 / 30)$ & $78.6(33 / 42)$ \\
& 1.22 & $94.1(16 / 17)$ & $72(18 / 25)$ & $69.6(16 / 23)$ & $94.7(18 / 19)$ & $81(34 / 42)$ \\
\hline
\end{tabular}

pos. value, positive predicative value; neg. value, negative predicative value.
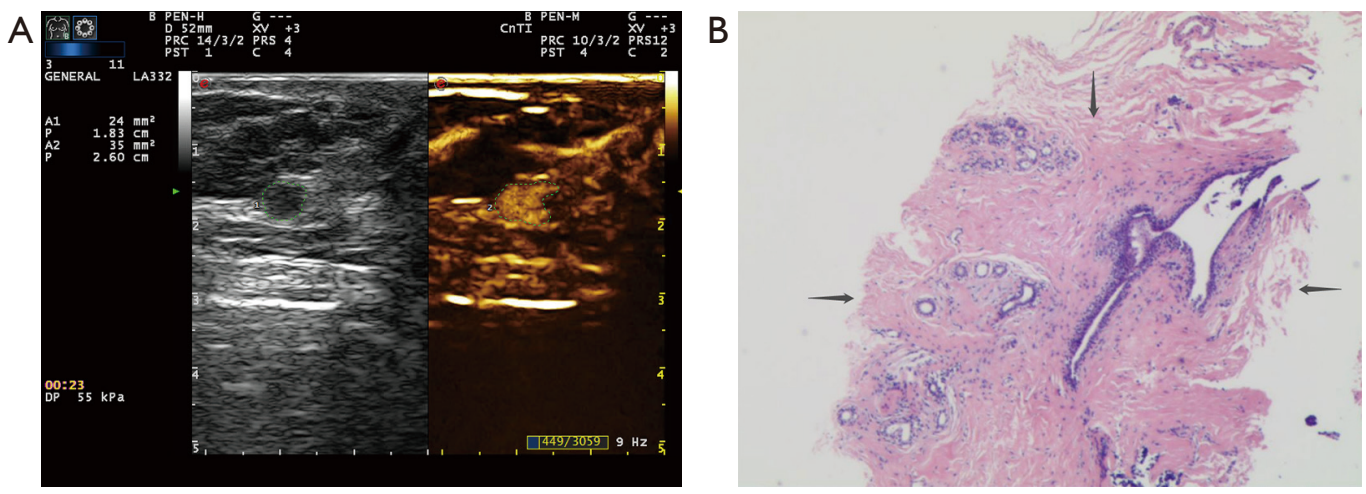

Figure 4 Benign breast mass was correctly assessed in a young patient before and after adjustment of the cutoff value. It came from a 28-year-old female patient. (A) Ultrasonography showed a hypoechoic mass in the left breast, the maximum diameter of the mass was 0.9 cm under conventional ultrasound, contrast area ratio $=1.38$; (B) pathological examination showed fibroadenoma (arrows) (HE $\times 40)$. When the cutoff value for diagnosis was 1.65 , the mass was assessed as benign mass, and when the cutoff value for diagnosis was increased to 1.95 , the mass was still diagnosed as benign mass.
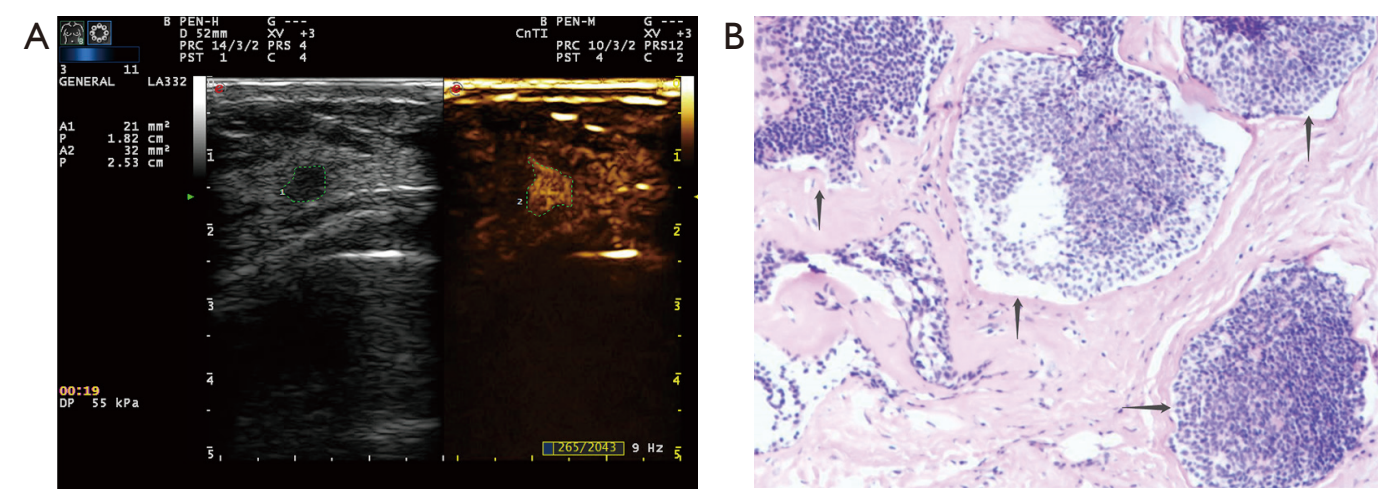

Figure 5 A malignant breast mass is correctly diagnosed in an old patient with a reduced cutoff value. It came from a 53-year-old female patient. (A) Ultrasonography showed a hypoechoic mass in the left breast, the maximum diameter of the mass was $0.6 \mathrm{~cm}$ under conventional ultrasound, contrast area ratio $=1.52$; (B) pathological examination showed ductal carcinoma in situ (arrows) (HE $\times 40)$. When the cutoff value for diagnosis was 1.65 , the mass was misjudged as a benign mass, but when the cutoff value for diagnosis was lowered to 1.22 , the mass was correctly diagnosed as malignant mass. 
obtained. When the cutoff value is reduced from 1.65 to 1.22 in elderly patients, the diagnostic sensitivity can be further improved without a significant reduction in specificity. Since the morphology and distribution of neovascularization in benign and malignant small breast masses overlap to a certain extent, in the differential diagnosis, a comprehensive evaluation should be performed by combining the medical history and other imaging methods, and the ultrasoundguided biopsy should be done when necessary to confirm the diagnosis.

\section{Acknowledgments}

Funding: None.

\section{Footnote}

Reporting Checklist: The authors have completed the STARD reporting checklist. Available at http://dx.doi.org/10.21037/ gs-20-697

Data Sharing Statement: Available at http://dx.doi. org/10.21037/gs-20-697

Conflicts of Interest: All authors have completed the ICMJE uniform disclosure form (available at http://dx. doi. org/10. 21037/gs-20-697). The authors have no conflicts of interest to declare.

Ethical Statement: The authors are accountable for all aspects of the work in ensuring that questions related to the accuracy or integrity of any part of the work are appropriately investigated and resolved. All procedures performed in this study involving human participants were in accordance with the Declaration of Helsinki (as revised in 2013). The study was reviewed and approved by the Medical Ethics Committee of the First Affiliated Hospital of Soochow University, with the written consent of all the patients taking part in the experiment.

Open Access Statement: This is an Open Access article distributed in accordance with the Creative Commons Attribution-NonCommercial-NoDerivs 4.0 International License (CC BY-NC-ND 4.0), which permits the noncommercial replication and distribution of the article with the strict proviso that no changes or edits are made and the original work is properly cited (including links to both the formal publication through the relevant DOI and the license).
See: https://creativecommons.org/licenses/by-nc-nd/4.0/.

\section{References}

1. Are C, Rajaram S, Are M, et al. A review of global cancer burden: Trends, challenges, strategies, and a role for surgeons. J Surg Oncol 2013;107:221-6.

2. Li YJ, Wen G, Wang Y, et al. Perfusion heterogeneity in breast tumors for assessment of angiogenesis. J Ultrasound Med 2013;32:1145-55.

3. Chen $Y$, Tang L, Du Z, et al. Factors influencing the performance of a diagnostic model including contrastenhanced ultrasound in 1023 breast lesions: comparison with histopathology. Ann Transl Med 2019;7:647.

4. de Vita R, Buccheri EM. Nipple sparing mastectomy and direct to implant breast reconstruction, validation of the safe procedure through the use of laser assisted indocyanine green fluorescent angiography. Gland Surg 2018;7:258-66.

5. Xiao X, Jiang Q, Wu H, et al. Diagnosis of subcentimetre breast lesions: combining BI-RADS-US with strain elastography and contrast-enhanced ultrasound-a preliminary study in China. Eur Radiol 2017;27:2443-50.

6. Balleyguier C, Opolonb P, Christine Mathieuc M, et al. New potential and applications of contrast-enhanced ultrasound of the breast: Own investigations and review of the literature. Eur J Radiol 2009;69:14-23.

7. Zhao H, Xu R, Ouyang Q, et al. Contrast-enhanced ultrasound is helpful in the differentiation of malignant and benign breast lesions. Eur J Radiol 2010;73:288-93 .

8. Liu H, Jiang Y, Dai Q, et al. Differentiation of benign and malignant sub-1-cm breast lesions using contrast-enhanced sonography. J Ultrasound Med 2015;34:117-23.

9. Xiao X, Ou B, Yang H, et al. Breast contrast-enhanced ultrasound: is a scoring system feasible? A preliminary study in China. PLoS One 2014;9:e105517.

10. Wang $Y$, Fan $W$, Zhao $S$, et al. Qualitative, quantitative and combination score systems in differential diagnosis of breast lesions by contrast-enhanced ultrasound. Eur J Radiol 2016;85:48-54.

11. Eugênio DS, Souza JA, Chojniak R, et al. Breast cancer features in women under the age of 40 years. Rev Assoc Med Bras (1992) 2016;62:755-61.

12. Mohey N, Hassan TA. Value of mammography and combined grey scale ultrasound and ultrasound elastography in the differentiation of solid breast lesions. The Egyptian Journal of Radiology and Nuclear Medicine 2014;45:253-61. 
13. Zuazo-Gaztelu I, Casanovas O. Unraveling the Role of Angiogenesis in Cancer Ecosystems. Front Oncol 2018;8:248.

14. Xiao XY, Chen X, Guan XF, et al. Superb microvascular imaging in diagnosis of breast lesions: a comparative study with contrast-enhanced ultrasonographic microvascular imaging. Br J Radiol 2016;89:20160546.

15. Li YJ, Wen G, Wang Y, et al. Perfusion heterogeneity in breast tumors for assessment of angiogenesis. J Ultrasound Med 2013;32:1145-55.

16. Jiang YX, Liu H, Liu JB, et al. Breast tumor size assessment: comparison of conventional ultrasound and contrast-enhanced ultrasound. Ultrasound Med Biol 2007;33:1873-81.

17. Metelková A, Skálová A, Fínek J. Breast Cancer in Young Women - Correlation of Clinical Histomorphological, and Molecular-genetic Features of Breast Carcinoma

Cite this article as: Wang $\mathrm{W}$, Zheng $\mathrm{Y}, \mathrm{Wu} \mathrm{XF}$, Zhao D, Hou LZ, Shi F, Liu JJ, Dong FL. Value of contrast-enhanced ultrasound area ratio in identifying benign and malignant small breast masses. Gland Surg 2020;9(5):1486-1494. doi: 10.21037/gs20-697 in Women Younger than 35 Years of Age. Klin Onkol. 2017;30:202-9.

18. Rudra S, Yu DS, Yu ES, et al. Locoregional and Distant Recurrence Patterns in Young versus Elderly Women Treated for Breast Cancer. Int J Breast Cancer 2015;2015:213123.

19. Liberman L, Morris EA, Dershaw DD, et al. Ductal enhancement on MR imaging of the breast. AJR Am J Roentgenol 2003;181:519-25.

20. Liu Y, Cao X. Immunosuppressive cells in tumor immune escape and metastasis. J Mol Med (Berl) 2016;94:509-22.

21. Jaffe TA, Martin LC, Thomas J, et al. Small-bowel obstruction: coronal reformations from isotropic voxels at 16-section multi-detector row CT. Radiology 2006;238:135-42.

(English Language Editor: J. Chapnick) 